\title{
Alternative Way of Recording Heart and Lung Sounds by a Modified Method in Veterinary Practice
}

\author{
Chinmoy Sarma ${ }^{1}$, Gautam Bordoloi ${ }^{2}$ and Sanjib Khargharia ${ }^{2 *}$ \\ ${ }^{1}$ Lakhimpur College of Veterinary Science, ${ }^{2}$ Lakhimpur College of Veterinary Science, \\ Assam Agricultural University, Lakhimpur, Assam, India \\ *Corresponding author
}

\section{A B S T R A C T}

\begin{tabular}{|l|}
\hline K e y w o r d s \\
$\begin{array}{l}\text { Proper diagnosis, } \\
\text { heart and lung } \\
\text { sounds, low-cost } \\
\text { device }\end{array}$ \\
\hline Article Info \\
\hline $\begin{array}{l}\text { Accepted: } \\
\text { 08 June } 2020 \\
\text { Available Online: } \\
\text { 10 July } 2020\end{array}$ \\
\hline
\end{tabular}

Diseases are the most crucial factor that reduces the productivity of animals. Proper diagnosis of diseases in field condition most of the time is difficult due to many factors. Auscultation of heart and lung sounds has vital role to play regarding the determination of health status of the animal. Since long, clinicians are dependent upon the use of stethoscope to detect the heart and lung sounds. This requires skill and experience, so to overcome this, a low-cost device is designed by using the chest piece of a stethoscope that is connected to a mobile phone and a sound is recorded which can be analysed later in the presence of experienced persons. Moreover, no specific mobile application is required for this. So, this costeffective device may be helpful for auscultation better way in field condition.

\section{Introduction}

Animal health is an important factor for the upliftment of rural agricultural economy. Diseases are major constraints in livestock sector that reduces the productivity of animals. Proper diagnosis of diseases in veterinary practice especially in the field condition is difficult many a times. The lung and heart sounds are considered as the most valuable tools that aids in proper diagnosis of the disease by a clinician. Obstruction of airways, emphysema, pneumonia, pulmonary oedema, bronchopneumonia, tracheal exudation etc. are some of the pulmonary disorders that leads to debilitating condition in animal resulting in reduced growth and productivity.

Moreover, most diseases related to an obstruction or restricted respiratory system or any aforesaid pulmonary condition is characterized from the sounds they generated while breathing. The auscultation is the most reliable technique and is also easy to perform for the detection of various pulmonary 
diseases of the respiratory system used by clinicians since old age (Sarkar et al., 2015). But in veterinary practice the diagnosis of respiratory diseases aided by auscultation techniques alone is doubtful most of the times (Charlotte, 2017).

Moreover, in veterinary field practices the optimal conditions for listening carefully the heart and lung sounds are difficult to get and to recognize the sounds and to gain proficiency in it is also difficult (Pedersen et al., 1999; Naylor et al., 2001; Ware, 2007). A simple stethoscope has been used till today for auscultation by the veterinary practitioner. In the present communication details of a simple handmade instrument for recording of heart and lung sound is mentioned by using stethoscope in modified method. This instrument might be helpful in the advancement of veterinary diagnostics where auscultation is difficult in normal way.

\section{The instrument proper}

An instrument is prepared by the author himself using a chest piece of a stethoscope along with its diaphragm and a microphone and a wire is connected with a $3.5 \mathrm{~mm}$ jack (Figure. 1). This is again connected to a mobile phone (Figure 2) and the sounds are recorded at the time of auscultation without installation of any kind of mobile applications. The instrument consists of a chest piece with diaphragm of stethoscope where the vibration from the heart and lung reaches. There is a $12 \mathrm{~cm}$ long tube fitted with diaphragmatic piece. This enables the sound wave propagated through the tube to reach the microphone. The microphone fitted to the other end of the tube which is directly connected to a mobile phone via a $3.5 \mathrm{~mm}$ jack to record the voice as such. The lung and heart sounds recorded with this instrument directly saved in the mobile phone which helps in farther analysis with the experts, health professionals of respective fields as well. Crackling, wheezing are abnormal lung sounds and muffled heart sound, murmur are abnormal heart sounds which can be directly recorded in the phone. This instrument can be made available at a minimum cost of production.

For authentication of accuracy of the equipment, two sets of respiratory and heart sounds were recorded from 8 numbers of animals of different species (Dog=2, cattle $=4$, goat=2) with the variation of time. The normal and abnormal lung sounds recorded are presented as frequency diagram in figure 3 and 4. On the other hand only the normal heart sound was recorded as available which is presented in figure 5 .

The graph shows the variation of the sounds. In normal lung sounds the peak point of the graph is in a regular interval and vibrations of sound in a continuous manner but when there is any abnormality in the sound like wheezing, crackling then the graphical vibration increases and irregular as shown in the figure 4 . The normal heart sound recorded in the present study is shown by a graph based on the vibrations of lub and dub.In lub and dub there is a definite gap of peak of the graph as shown in the figure 5.

Lung and heart sounds are the important parameter for any diagnosis of disease and also to know whether the animal cope up with introduction of heavy dose of medicine or not. When the recorded heart and lung sounds looked into by some experts before onset of any surgical procedure with or without any anaesthetic protocol then it is easier to perform a smooth surgery as well as to prevent any complications during surgery. 


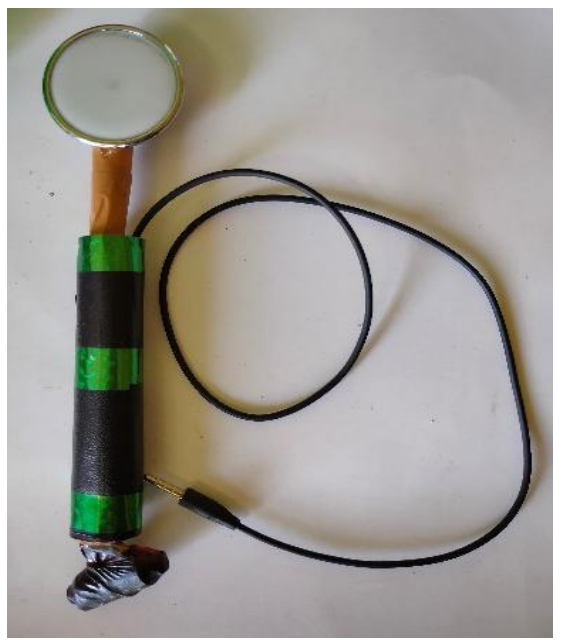

Figure.1 Diaphragm with $12 \mathrm{~cm}$ tube connected to a wire with $3.5 \mathrm{~mm}$ jack

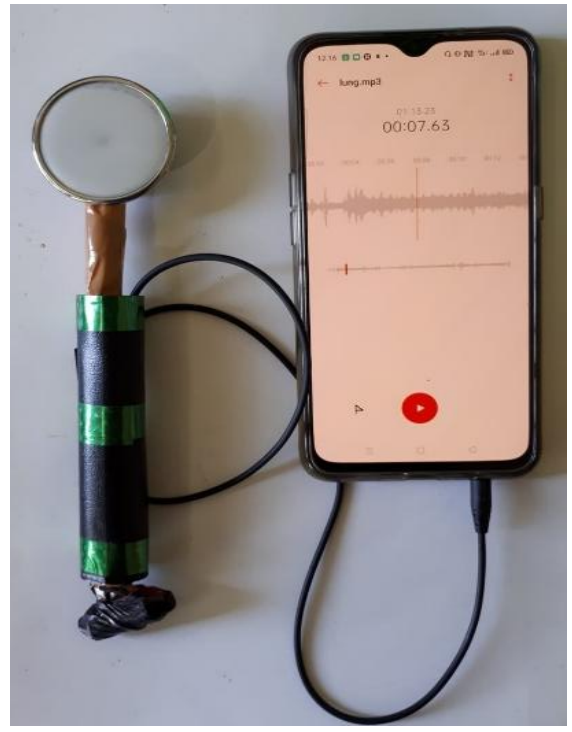

Figure.2 Instrument connected with mobile phone

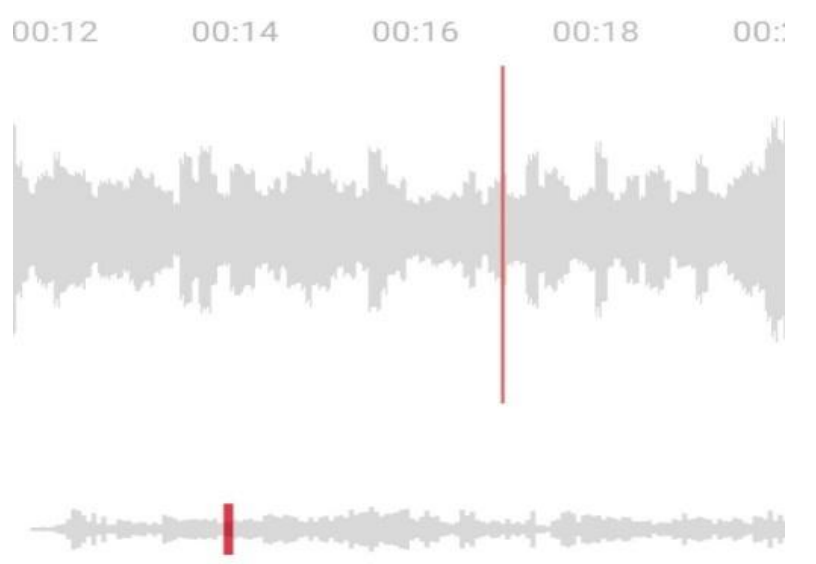

Figure.3 Normal lung sound recorded shown by the graph (represented) 


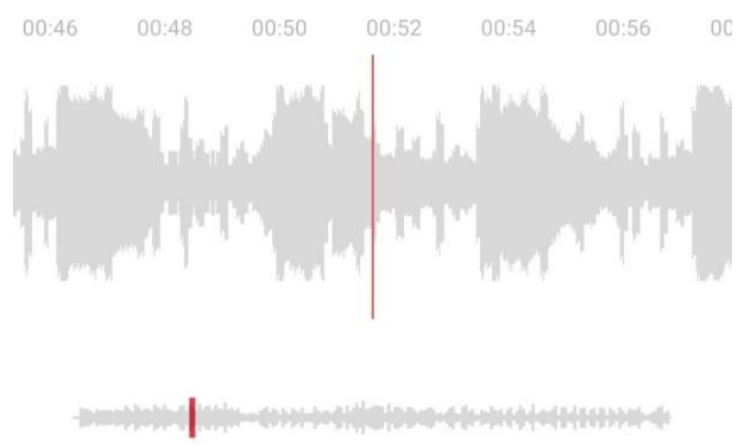

Figure.4 Abnormal lung sound (expiratory wheezing) shown by the graph (represented)

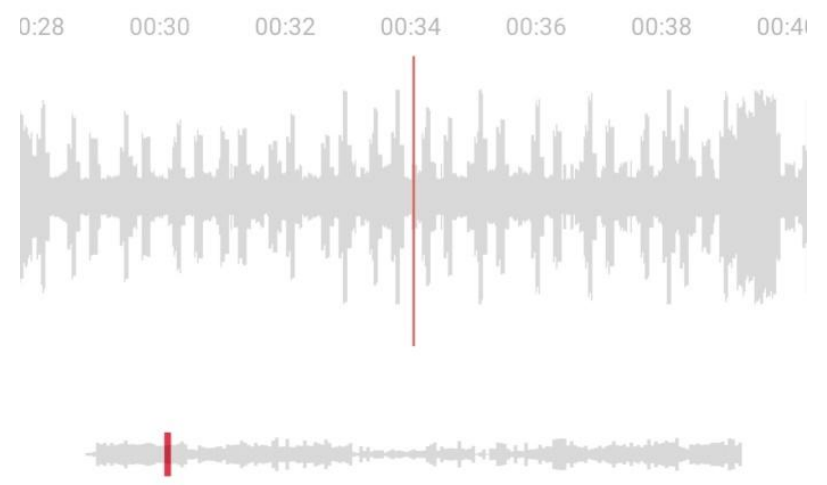

Figure.5 Normal heart sound vibrations

As this instrument does not require any mobile application unlike the one which was made for human practices where smart phone and mobile applications were used to analyze the recorded sounds (crackling lung sound) (Reyes et al., 2018), it can be operated in any phone without downloading any application program. There is no other such type of known instrument available for auscultation of heart and lung sound till now in veterinary practice. This might be a valuable instrument for veterinary practitioners as it is very much flexible and can be easily carried to the field. Moreover, it can be made easily in low cost or the clinician by their own.

This instrument will help veterinary practitioners to deal with the patients as the instrument can be carried easily to the field and can be made with a minimum cost. In veterinary practice, clinicians sometime have to face lack of assistance in the field for monitoring of heart and lung sound which is essential during surgical operation. This is the first approach made in the field of veterinary practice to find alternative, easier and costeffective way in diagnostics as per the available literatures available. This instrument will be a boon to the veterinary profession at a large to maintain a healthy animal society by giving accurate diagnosis and proper treatment.

\section{References}

Bersain A. Reyes, Nemecio Olvera-Montes, Sonia Charleston-Villalobos, Ramón González-Camarena, Mayra Mejía- 
Ávila and Tomas Aljama-Corrales (2018). A Smartphone-Based System for Automated Bedside Detection of Crackle Sounds in Diffuse Interstitial Pneumonia Patients. Sensors, 18, 3813.

Charlotte Pace (2017). How to maximise your auscultation technique. The Veterinary Nurse, 8(9):512-515).

Malay Sarkar, Irappa Madabhavi, Narasimhalu Niranjan, and Megha Dogra (2015). Auscultation of the respiratory system. Ann Thorac Med., 10(3): 158-168.

Naylor JM, Yadernuk LM, Pharr JW, Ashburner JS (2001). An assessment of the ability of diplomates, practitioners, and students to describe and interpret recordings of heart murmurs and arrhythmia. J Vet Intern Med. 15(6):507-15.

Pedersen HD, Häggström J, Falk $\mathrm{T}$ et al., (1999). Auscultation in mild mitral regurgitation in dogs: observer variation, effects of physical maneuvers, and agreement with color Doppler echocardiography and phonocardiography. J Vet Intern Med. 13(1):56-64.

Rynae E Englar (2019). Muffled Heart Sounds. Common Clinical Presentations in Dogs and Cats, 561567.

doi:10.1002/9781119414612.ch42)

Ware W (2007). Cardiovascular Disease in Small Animal Medicine. Manson Publishing, London

\section{How to cite this article:}

Chinmoy Sarma, Gautam Bordoloi and Sanjib Khargharia. 2020. Alternative Way of Recording Heart and Lung Sounds by a Modified Method in Veterinary Practice. Int.J.Curr.Microbiol.App.Sci. 9(07): 856-860. doi: https://doi.org/10.20546/ijcmas.2020.907.099 